\title{
Burnout and Turnover Intention Among Peer Providers and Other Providers of Mental Health Services in a Rural Two-County Area
}

\author{
Kim Weikel[D - Thomas Fisher
}

Received: 4 May 2021 / Accepted: 12 August 2021/Published online: 24 August 2021

(C) The Author(s), under exclusive licence to Springer Nature India Private Limited 2021

\begin{abstract}
Peer providers in the mental health field, having their own lived experience with mental illness, use their personal experience to assist the consumers of their services. Given high burnout rates in the mental health field, there have been two prior investigations of burnout among peer providers. The present study extends those prior investigations to a sample of peer providers working in community mental health programs in a rural area of the United States. Consistent with the prior studies, there were no differences in burnout between peer providers and other providers employed by those programs, with particularly strong support for the null hypothesis regarding the emotional exhaustion component of burnout. There were also no differences in strength of turnover intentions. However, among the non-consumer providers emotional exhaustion was correlated with both types of turnover intention assessed in this study (intent to change job setting within the mental health field and intent to leave the mental health workforce), whereas among the peer providers emotional exhaustion was correlated only with intent to leave the mental health field. Workload dissatisfaction was the workplace variable most strongly associated with emotional exhaustion among both types of providers. Other moderately strong inverse predictors
\end{abstract}

K. Weikel $(\varangle) \cdot$ T. Fisher

Psychology Department, Shippensburg University of

Pennsylvania, Shippensburg, PA, USA

e-mail: kaweik@ship.edu of emotional exhaustion among peer providers were workplace community and workplace control, and the correlation between emotional exhaustion and workplace control was significantly stronger among the peer providers than among the other providers.

Keywords Burnout - Peer providers - Turnover · Mental health $\cdot$ Rural

\section{Introduction}

The Recovery-oriented model for mental health services recognizes mental health recovery as a nonlinear process that goes beyond symptom reduction and can be meaningfully pursued even when there are ongoing symptoms. Peer support is recognized as an important component of mental health recovery [37], and there has been growing enthusiasm for employing mental health consumers, or peer providers, in a variety of positions within the mental health field $[8,24]$. Peer providers, having their own lived experience with mental illness, intentionally use their personal experience to assist the recipients of their services as they continue to pursue and maintain their own recovery.

Holding a variety of positions in either consumerrun or traditional clinical or rehabilitation programs, peer providers have a unique ability to offer hope by 
disclosing accounts of their personal struggles, discussing their successful recovery strategies, or imparting knowledge gained through their own successful navigation of the mental health system (e.g., [9, 24]). Such intentional use of personal experiences is known to be helpful, as peer support has been associated with reduced hospitalizations (e.g., [11, 36]), increased treatment engagement [35], and improvements in other mental health recovery areas like hopefulness and self-advocacy [4]. Furthermore, provision of peer support can also be rewarding for the providers. Providing peer support is correlated with higher selfesteem [3], and peer providers have reported improvements in their personal mental health recovery through their provision of services [22]. Concurrent with the benefits of peer provider positions, however, is worry that the continued recovery of peer providers could be interrupted because of stress in such a high burnout field. Reviews of multiple studies have illuminated the high rates of burnout in the mental healthcare work force, [10, 27].

Burnout in the human services field can involve three components: feelings of emotional exhaustion, depersonalization of clients, and a low sense of personal accomplishment [20]. Estimates (preCOVID-19) of the percentage of mental healthcare workers experiencing high burnout have ranged from 21 to $67 \%$ [23], and burnout rates may be particularly high among those providing services for individuals with serious and persistent mental illness [1]. Peer providers offer services primarily, sometimes exclusively, for individuals with serious mental illness (e.g., [4]), placing them in that high-risk group. On the other hand, burnout is lower among mental health providers working in more recovery-oriented systems [14] and among workers who can relate to their clients and encourage a sense of empowerment [38], variables that describe the work of peer providers. Thus, some work-related variables may buffer the rate of burnout among peer providers.

Recognizing the uncertain potential for burnout among peer providers, Park and colleagues [29] examined burnout rates among 152 peer support specialists within the Veterans Health Administration (VHA) system across a 12-month period. Finding no significant differences in burnout rates among peer providers compared to other mental health workers in the VHA system, and that the number of hours providing direct client services was not a predictor of burnout at either baseline or one year later, they concluded that peer providers are no more susceptible to burnout than other mental health employees. In addition, although the correlation between burnout and psychiatric symptom severity found among other mental health workers (e.g., [5]) was also present among the peer providers, greater symptom severity predicted lower depersonalization among the peer providers one year later, leading Park and colleagues (2016) to suggest that greater symptom severity among peer providers might result in stronger empathy for clients.

The Veterans Health Administration (VHA) system is recognized as a leader in instituting recoveryoriented care and employing peer workers [24]. Thus, the VHA data, demonstrating that burnout rates among their peer support specialists were no higher than those of their other mental health services employees, is important and promising. However, generalization of their results is unknown, leaving one to wonder whether burnout could be stronger among peer providers working in other settings such as smaller community agencies. For example, workplace stressors such as role confusion, a common complaint among peer supporters (e.g., [21]), might be stronger in community systems offering peer support programs that are less well established than the VHA programs, and role confusion might result in stronger emotional exhaustion and lower personal accomplishment. Furthermore, peer providers in smaller community agencies may hold more general positions within the mental health field, rather than holding the more specific positions of peer supporter or peer specialist, and therefore may experience less support than those employed in jobs specifically designated as peer support positions. Consequently, investigating burnout rates among peer workers employed by smaller community mental health organizations will be important before confidently concluding that burnout rates are not higher among peer providers.

A recent study in Australia found no differences in burnout rates between peer workers and other mental healthcare workers, and no differences between peer providers working for government managed programs as compared to those in community managed programs [33]. The present study, sparked by a request from a county mental health system, involved estimating rates of burnout among peer and non-consumer employees of county-contracted small community 
mental health programs in a rural two-county area in the United States. Early discussion with supervisors from one of the programs elucidated a concern that burnout among mental health providers could be a partial explanation for employee turnover. Such concern is consistent with previously noted associations between burnout and turnover (e.g., [28]), including a recent COVID-19-related study demonstrating associations between burnout and turnover intention among providers in community mental health centers [34] as well as an earlier study demonstrating association between turnover intention and emotional exhaustion among peer providers in Australia [33]. Thus, the present study included assessment of intent to leave one's job.

In addition, the present study also explored possible predictors of burnout. Although there are indications that burnout rates among peer providers are no higher than those of other providers, the mental healthcare field is still left with high burnout rates in general and an obvious need to address burnout, a condition that is associated with factors such as depression and anxiety [13], poorer physical health [1,30], sick days [28], and self-reported recognition of providing lower quality care [32]. Based on an extensive review of research regarding burnout among mental healthcare workers, Johnson and colleagues (2017) have recommended that burnout interventions address both person-level and agency-level variables. Consequently, the present study included assessment of self-compassion, a person-level variable that has been the focus of recent burnout intervention programs (e.g., [26]), and assessment of work life variables purported to be possible predictors of burnout [16].

Self-compassion involves self-kindness rather than self-judgment, an understanding of common humanity, and a mindful openness to painful experience [25]. Mental healthcare workers with higher levels of selfcompassion have lower levels of burnout [2], and training in mindful self-compassion can reduce burnout among professionals in the healthcare field $[6,26]$. Given the possibility that initially confident peer providers may be susceptible to burnout when they are later faced with slow progress among some of their peers or with frustrations related to barriers in the field (see [29]), and that self-compassion in reaction to such disappointments might protect against burnout, the present authors wondered whether the association between self-compassion and burnout might be especially strong among peer providers.

Similarly, some work life variables may be particularly strong predictors of burnout among peer providers. Leiter and Maslach [15], leaders in the assessment of burnout, developed the Areas of Work Life Scale (AWS) to measure congruency between employees' work lives and their personal expectations regarding work experiences in six areas they believe to be associated with burnout: workload, control, reward, community, fairness, and values. We wondered whether one of those areas, workplace community, might be an especially strong predictor of burnout in peer providers. Among peer providers in Australia, workplace culture and attitudes of clinicians was the most frequently reported negative aspect of their jobs [33]. Similarly, interviews with peer providers have found them to report poor treatment in the workplace [18], with more than half reporting experiences of direct and indirect prejudice relating to their peer provider status [21] as well as microaggressions involving negative comments and invalidating messages about the role of peer support [7]. Thus, the present authors predicted that scores on the AWS subscale measuring sense of Community might be the strongest predictor of emotional exhaustion among peer providers. In addition, given that peer providers are likely to strongly value the recovery-oriented model for mental health services, but that some peers find themselves in settings that seem to lack a recovery-oriented philosophy [21], it was also hypothesized that the AWS subscale measuring person-work Values discrepancies would be a stronger predictor of burnout among peer providers than among nonconsumer workers. Of course, although dissatisfaction with community interactions and with the values of the workplace may occur among many peer supporters, it is noteworthy that some peer providers work in agencies known as consumer-run or peer-run programs, programs which are naturally recovery-oriented and in which most employees are peer providers. Exploring differences between those two peer provider workplace environments (traditional mental health programs and peer-run programs), when possible, may be helpful. The present investigators were able to do some beginning-level explorations of possible differences between peer provider experiences in those varying workplace settings. 
In summary, the present study was an attempt to replicate previous findings $[29,33]$ of no differences in burnout rates between peer providers and non-consumer providers, extending those results to community-based mental healthcare providers in a small rural area of the U.S. The authors also explored associations between burnout and turnover intention as well as associations between burnout and possible predictor variables, hypothesizing that self-compassion might be an especially strong buffer against burnout among peer providers and that two work life variables (dissatisfaction with workplace community interactions and dissatisfaction with workplace values) might be more strongly associated with burnout among peer providers than among non-consumer providers.

\section{Method}

Participants and Procedure

Participants were 74 mental health workers who volunteered to complete an anonymous survey regarding their work experiences. All workers were employed by agencies with county contracts to provide mental health services for individuals residing within a rural two-county area in Pennsylvania. Fiftysix participants took the survey on-line (via Survey Monkey) by clicking a link in an e-mail forwarded to them by an agency representative. There were no restrictions on when or where they could take the survey. One of the agencies, a peer-run agency providing multiple peer support services in the twocounty area, reported limited access to computers among their employees and requested a paper-andpencil version of the survey; 18 of their employees completed a paper-and-pencil version (printed directly from the Survey Monkey program) at the start of a training meeting. Data were gathered from 3/21/2019 to $12 / 4 / 2019$ (and, therefore, represent pre-COVID-19 levels of burnout and turnover intention). The survey and methodology were approved by the Shippensburg University Institutional Review Board/Human Subjects Review Committee (Ethics approval numbers: 02-14-19 Weikel \#1448 - TW and 08-30-19 Weikel 1777 - TW). Informed consent was indicated through an item on the online survey and was indicated on a page, collected separately, when the paper-and-pencil version was completed. All survey responses were anonymous, and possibly identifying demographic information was stored separately from other survey responses.

Most of the participants were female $(82 \%)$ and $90 \%$ were White. Participants were considered peer providers for this study if they responded yes to the question "do you have lived experience with your own diagnosed mental illness and use that first-hand experience to assist your work with consumers/clients?" One participant did not respond to the question. Of the remaining 73 participants, 38 $(52.1 \%)$ were peer providers. Experience working in the mental health field ranged from 1 to 35 years for the peer providers and less than six months to 45 years for the non-consumer providers. Seventy-three percent of the peer providers and seventy-seven percent of the non-consumer providers reported having at least a bachelor's degree, and there were both peer providers and non-consumer providers working in inpatient, outpatient, case management, in-home/mobile, crisis, and social rehabilitation/drop-in programs.

\section{Measures}

\section{Burnout}

Burnout was measured with the Maslach Burnout Inventory- Human Services Survey (MBI-HSS; [19]), which defines burnout in terms of three dimensions: emotional exhaustion, depersonalization, and sense of personal accomplishment. Considered the gold standard for assessing burnout, the MBI has clear indications of reliability (with reliability estimates, across multiple studies, generally in the high $0.80 \mathrm{~s}$ for emotional exhaustion and in the mid-70 $\mathrm{s}$ for the depersonalization and the personal accomplishment subscales, but with estimates occasionally in the $0.50 \mathrm{~s}$ for the depersonalization scale) and multiple validity indicators [20]. In the present study, internal consistency for emotional exhaustion was 0.91 among the peer providers and 0.94 among the non-consumer providers and was above 0.80 for personal accomplishment in both groups of providers. Internal consistency for the depersonalization scale was 0.73 among the non-consumer providers but 0.65 among the peer providers. 
Self-Compassion

Self-compassion was measured with the short-form version of the Self-Compassion Scale (SCS-SF; [31]). This 12-item scale includes items representing each of the original SCS subscales of Self-Kindness, SelfJudgement, Common Humanity, Isolation, Mindfulness, and Over-Identification. It has good internal consistency $(\alpha=0.87)$ and correlates 0.97 with the original longer version, a scale with strong reliability and validity [25, 31]. Internal Consistency in the present study was 0.90 among the peer providers and 0.85 among the non-consumer providers.

\section{Work Environment}

Work environment factors that might be associated with burnout were measured utilizing the Areas of Worklife Survey (AWS; [15]). The AWS includes 28 likert-scale items divided into six subscales: workload, control, reward, community, fairness, and values. Scores on each subscale represent the extent to which there is good fit between the work environment and an employee's personal expectations for that work area, with higher scores indicating better fit. Internal consistency estimates range from 0.67 to 0.83 , testretest correlations range from 0.51 to 0.62 for the subscales, and correlations between each AWS subscale and related complaints from over 1000 hospital employees indicate subscale validity [16]. Internal consistency was above 0.75 on all subscales for both the peer and non-consumer providers in the present study.

\section{Turnover Intention}

Turnover intention was measured with two questions: "how likely are you to look for a new job in the field of mental health within the next year" and "how likely are you to look for a new job outside the field of mental health within the next year." Participants responded using a 5-point scale (with responses ranging from not at all likely to very likely). Each question was analyzed separately, and higher scores indicate higher turnover intention.

\section{Results}

Data analyses were completed using IBM SPSS Statistics 27, and missing data were handled by excluding cases on an analysis-by-analysis manner. All participants completing the paper-and-pencil version of the survey were employed by a peer-run program, and all but two of those participants reported being a peer provider. In contrast, none of the online survey participants reported working for a peer-run program. Thus, $39.5 \%$ of the peer providers differed from other peer providers in two ways, having completed a paper-and-pencil version of the survey and working for a peer-run organization. For this reason, analyses began with t-tests investigating possible differences between the two groups of peer providers on all measures (See Table 1). The only statistically significant difference was on depersonalization scores, with peer providers in the peer-run program who completed the paper-and-pencil survey reporting significantly less depersonalization of clients $(M=1.60)$ than the peer providers who do not work for peer-run programs and completed the online survey $(M=4.05)$. Given little indication of other differences due to survey format or peer-run work setting (the only other difference approaching significance, $p=0.08$, was for control) the peer provider groups were collapsed and t-tests were completed to determine differences between peer providers and non-consumer providers.

There were no statistically significant differences between peer and non-consumer providers on any of the measures (see Table 2). We followed the $t$-tests with Bayesian analyses of differences between independent groups to estimate the probability of the null hypothesis (i.e., no differences in burnout between peer and non-consumer providers). The Bayes factor (Rouder method; null vs. alternative) for emotional exhaustion $(\mathrm{BF}=4.76)$ indicated odds of $4: 1$ in favor of the null hypothesis that there were no differences in emotional exhaustion between the two types of providers; Bayes factors above 3 can be interpreted as indicating positive evidence for the null hypothesis [12]. Bayes Factors indicated weaker support for the null hypothesis regarding depersonalization and personal accomplishment.

The Emotional Exhaustion MBI subscale is a particularly reliable indicator of burnout, and peer providers in the present study, regardless of whether 
Table 1 Comparisons between peer providers in peer-run programs and other peer providers

\begin{tabular}{lllr}
\hline Variable & \multicolumn{2}{l}{ Mean (SD) } & \multirow{2}{*}{$t$} \\
\cline { 2 - 3 } & Peer-run & Other & \\
\hline Emotional exhaustion & $17.60(11.53)$ & $18.95(11.77)$ & 0.346 \\
Depersonalization & $1.60(1.99)$ & $4.05(3.50)$ & $2.43^{*}$ \\
Personal Accomplishment & $37.29(9.39)$ & $41.37(5.17)$ & 1.60 \\
Turnover intent (within mental health) & $2.44(1.46)$ & $2.11(1.45)$ & -0.67 \\
Intent to leave mental health field & $2.63(1.50)$ & $1.95(1.18)$ & -1.50 \\
Workload Congruency/Satisfaction & $3.26(1.06)$ & $3.11(0.92)$ & -0.45 \\
Control & $4.05(0.65)$ & $3.60(0.80)$ & -1.79 \\
Reward & $3.47(0.92)$ & $3.67(0.95)$ & 0.64 \\
Community & $3.73(0.62)$ & $3.77(0.73)$ & 0.19 \\
Fairness & $3.67(0.45)$ & $3.35(0.80)$ & -1.41 \\
Values & $4.03(0.49)$ & $3.82(0.73)$ & -1.00 \\
Self-Compassion & $3.13(0.77)$ & $3.17(0.86)$ & 0.15 \\
\hline
\end{tabular}

Table 2 Comparisons between peer providers and non-consumer providers

\begin{tabular}{lllll}
\hline Variable & \multicolumn{1}{l}{ Mean (SD) } & \multirow{2}{*}{ Bayes Factor $^{a}$} \\
\cline { 2 - 4 } & Peer providers & Non-consumer providers & & \\
\hline Emotional exhaustion & $18.58(11.64)$ & $20.23(12.38)$ & -0.58 & 4.76 \\
Depersonalization & $3.00(3.16)$ & $4.88(5.14)$ & -1.84 & 1.01 \\
Personal accomplishment & $39.64(7.42)$ & $36.97(6.91)$ & 1.51 & 1.91 \\
Turnover intent (within MH) & $2.26(1.44)$ & $2.00(1.00)$ & 0.83 & 3.24 \\
Intent to leave MH field & $2.26(1.36)$ & $1.84(1.14)$ & 1.34 & 2.16 \\
Workload satisfaction & $3.18(0.97)$ & $3.11(0.93)$ & 0.30 & 5.27 \\
Control & $3.81(0.75)$ & $3.57(0.96)$ & 0.39 & 3.46 \\
Reward & $3.58(0.93)$ & $3.49(0.88)$ & -0.14 & 5.13 \\
Community & $3.75(0.67)$ & $3.77(0.81)$ & 1.50 & 5.31 \\
Fairness & $3.50(0.67)$ & $3.23(0.78)$ & 0.74 & 1.95 \\
Values & $3.91(0.63)$ & $3.78(0.84)$ & -0.71 & 4.17 \\
Self-Compassion & $3.15(0.81)$ & $3.28(0.63)$ & & 4.29 \\
\hline
\end{tabular}

${ }^{a}$ Bayes Factors are for null vs. alternative

they worked for a peer-run or other type of program, reported similar levels of emotional exhaustion. Consequently, like some other prior studies of burnout in the mental healthcare field (e.g., [39]), the present authors used emotional exhaustion scores as an indicator of burnout for additional analyses.

Turnover Intention

Among the peer providers, there was a near-zero correlation $(r=-0.062)$ between emotional exhaustion and intention to look for a different job within the mental health field. In contrast, there was a statistically significant correlation $(r=0.488)$ between their emotional exhaustion and their intention to look for a new job outside the mental health field. Among the non-consumer providers, emotional exhaustion was significantly associated with turnover intention both within and outside the mental health field. 
Table 3 Correlations (r) with the emotional exhaustion (EE) subscale of the $\mathrm{MBI}$ and $\mathrm{r}$ to $\mathrm{Z}$ transformations to test differences in strength of $r$

${ }^{*} p<.05, * * p<.01$ $* * * p<.001$

\begin{tabular}{llll}
\hline Variable & Peer Providers & $\begin{array}{l}\text { Non-consumer } \\
\text { Providers }\end{array}$ & $Z$ \\
\hline Workload congruency/satisfaction & $-.670^{* * *}$ & $-.525^{* *}$ & -0.87 \\
Control & $-.551^{* *}$ & -.164 & $-1.73^{*}$ \\
Reward & $-.482^{* *}$ & $-.464^{* *}$ & -0.09 \\
Community & $-.540^{* *}$ & -.243 & -1.36 \\
Fairness & -.210 & -.098 & -0.44 \\
Values & -.333 & -.148 & -0.76 \\
Self-Compassion & $-.429 *$ & -.287 & -0.63 \\
Turnover intent (within mental health) & -.062 & $.357^{*}$ & $-1.66^{*}$ \\
Intent to leave mental health field & $.448^{* *}$ & $.390^{*}$ & 0.27 \\
\hline
\end{tabular}

\section{Discussion}

The present study offers further support for a conclusion that mental health peer providers are no more likely to experience burnout than non-consumer providers. The results extend the prior findings from the VHA system, providing evidence of generalization to a small community sample. Thus, the present authors echo the conclusion of Park and colleagues [29] that the inclusion of peer support workers in the mental health field can be done without worry that they are more susceptible to burnout.

This, of course, does not mean that peer providers should not be offered support. Although peer providers do not appear to be more susceptible to burnout, there is certainly reason to address the high rates of burnout among all mental health providers. Beyond the obvious reasons for attempting to alleviate emotional distress associated with working in the mental health field, reducing turnover is another important reason for pursuing burnout prevention and intervention. Similar to the findings of prior studies (e.g., 33 , 39]), emotional exhaustion was significantly correlated with turnover intention among both the peer and the non-consumer providers.

Li and colleagues [17] have suggested that not all types of turnover intentions are alike and recommend distinguishing between intent to change jobs within the same field from intent to switch occupations. In this sample, emotional exhaustion was associated with both types of turnover intentions among the nonconsumer providers. Thus, non-consumer providers experiencing higher levels of emotional exhaustion may consider moving to different agencies within the mental health field in addition to considering total 
occupational changes. In contrast, among the peer providers there was a near zero correlation between emotional exhaustion and intention to change jobs within the mental health field but a moderately strong significant correlation between emotional exhaustion and intent to leave the mental health workforce. Thus, the mental health field may be more likely to lose peer providers entirely when those providers are experiencing higher levels of burnout.

We anticipated that there might be differences in the specific types of factors associated with burnout among peer providers as compared to non-consumer providers. Highlighting the factors particularly associated with burnout among peer providers could help to refine burnout prevention and intervention efforts. One clear finding is that workloads exceeding workers' perception of being accomplishable are strongly associated with burnout among both peer and nonconsumer providers. Dissatisfaction with workload was the workplace variable most strongly associated with burnout among both types of providers, and the strong correlation between workload dissatisfaction and burnout is a finding consistent with prior research [16]. Clearly there is a need to address workload as part of burnout prevention efforts.

Given reports of microaggressions and other indications of discrimination and hostile comments from fellow employees directed toward peer providers (e.g., 7 ), it was predicted that dissatisfaction with workplace community might be more strongly associated with burnout among the peer providers than among their non-consumer counterparts. Although there was a significant association between community and emotional exhaustion among the peer providers, that association was not significantly stronger than the correlation between community and emotional exhaustion among the non-consumer providers. It is important to note that the community subscale of the AWS does not distinguish between various types of community dissatisfaction and may not have captured the specific types of distressing interactions that could be strong predictors of burnout among peer providers. Consequently, given the moderately strong $(r=0.54)$ correlation between workplace community and emotional exhaustion among the peer providers in the present study, we recommend further investigation of that association and suggest advancing the work of Furmin and colleagues [7] by investigating possible association between burnout and the more specific variable of workplace microaggressions.

Also tested was the hypothesis that discrepant workplace values might be a particularly strong predictor of burnout among peer providers, but the weak correlations between workplace values and emotional exhaustion were not statistically significant among either the peer or non-consumer providers in this small sample. Given that the values AWS subscale is not specific to values discrepancies related to recovery-oriented philosophy, future researchers may find it helpful to explore the possibility that dissatisfaction with the recovery-oriented integrity of their employers may be a specific predictor of burnout among peer providers.

What was not predicted was the difference between peer and non-consumer providers in the importance of workplace control. The moderately strong correlation between emotional exhaustion and workplace control among the peer providers was significantly stronger than the weak, nonsignificant correlation among the non-consumer providers. The control subscale on the Areas of Worklife Survey encapsulates the degree to which employees believe they can influence decisions that have an impact on their work [16]. There are several possible sources of a reduced sense of control in the workplace, such as the role conflict or authority issues that could be experienced by all types of service providers. Another type of reduced sense of control can result from role ambiguity, involving uncertainty regarding the expectations of one's position, which can result in difficulty establishing what one should strive to accomplish [16]. Such role ambiguity is a frequently reported complaint among peer providers (e.g., [21]), and it has been suggested that clearer job descriptions may enhance the work of peer supporters and provide further validation for the peer supporter position [9]. Future researchers may want to investigate associations between role ambiguity and burnout among peer providers, as such associations may be one more reason to strive for clearer descriptions of the peer supporter role.

As we predicted based on prior findings for other mental healthcare workers [2], there was a statistically significant negative correlation between self-compassion and emotional exhaustion among the peer providers. Self-compassion may play a buffering role against emotional exhaustion among peer providers. Given prior support for the helpfulness of self- 
compassion training for reducing burnout $[6,26]$, such training may be an effective component for burnout interventions and prevention efforts for peer providers.

The present study included exploratory analyses of differences between peer providers employed by peerrun programs and those who have been hired by mental health programs employing both peer and nonconsumer workers. It is possible that work life atmosphere for providers in peer-run programs could differ from the atmosphere experienced by peer providers in other programs, resulting in varying experiences with job satisfaction and even burnout. However, we found no differences between peer providers in peer-run vs traditional programs in emotional exhaustion, sense of personal accomplishment, or intent to leave the job, and no differences in the work life variables included on the AWS. This finding offers beginning support for a suggestion that not only are peer providers no more susceptible to burnout than non-consumer providers, but that peer providers working amidst non-consumer providers in traditional mental health programs are no more susceptible to emotional exhaustion than those working for peer-run programs. Of course, our sample included only one peer-run program so there is clearly a need for future investigations before making such claims with confidence.

The variable for which we did find a difference between peer providers in peer-run programs and those in traditional programs was depersonalization, with peer providers in peer-run programs reporting significantly less depersonalization of their peer clients. However, it is difficult to interpret the reason for that difference. While it is possible that individuals working for peer run programs are the providers most likely to hold especially strong recovery-oriented philosophy and to be least likely to withdraw from their connection with fellow consumers, it is equally likely that the differences could be due to the paperand-pencil group-administered format of the survey which was completed exclusively by the peer providers in the peer-run program, a format which may have discouraged affirmative responses to the socially undesirable questions regarding depersonalization of clients. It is also possible that the difference is due to something unique to that one particular peerrun program. The difference is intriguing, however, especially considering the Park et al. [29] suggestion that greater symptom severity among peer providers might be associated with less depersonalization and greater empathy for peer consumers. The present authors wonder whether such an association might be particularly strong among peers working in peer-run programs which are likely to have an especially strong recovery-oriented focus. Further exploration of possible differences between peer providers in peer-run programs and peer providers in other mental health programs is recommended.

Clear limitations of the present study are the small sample size and the lack of racial diversity among the participants. Although this study is meant to support generalization of findings from studies with larger sample sizes, the small and racially homogeneous sample limits the confidence with which the authors can draw conclusions. The sample was also limited to a two-county area in rural Pennsylvania. Multiple future replications of this study in varied small community settings could be helpful, as could a larger-scale survey of multiple small community programs.

In addition, the present findings are based entirely on quantitative analyses using pre-established measures. As mentioned by a reviewer of this study, qualitative analysis of interviews with both peer and non-consumer providers could help to elucidate some of the results. For example, such interviews could be especially helpful for identifying the types of dissatisfaction with community and workplace control associated with burnout among peer providers, and interview responses might also identify some experiential differences between peer providers working in traditional programs and those working in peer-run programs.

It is also important to note that the definition of peer provider in the present study may have been broader than that of prior studies. Peer providers (who reported that they have lived experience with mental illness and use that first-hand experience to assist their work with consumer/clients) were not asked whether they regularly disclose their peer status to their clients. Thus, it is possible that some of the peer providers in this study, who may make more covert use of their peer status, would not have been considered peer providers in other studies. Although this study provides information about burnout rates among all peers working in the mental health field, a narrower definition of peer 
provider may enhance the ability to compare results across studies.

In summary, the present study provides further support for the conclusion that peer providers are no more susceptible to burnout than other mental healthcare providers. In addition, there is beginning-level suggestion that peer providers working in traditional mental health programs are no more susceptible to emotional exhaustion than those working for peer-run programs. Although there was little evidence of differences in the types of factors associated with burnout among peer providers and non-consumer providers, this study did not include assessment of workplace variables that may be more specific to peer providers. For example, the association between burnout and predictors such as workplace microaggression and workplace role ambiguity may be especially strong among peer providers, and future investigations of such associations, utilizing both standardized surveys and open-ended interviews, may assist with fine tuning burnout prevention efforts specifically for peer providers.

Funding This study was sponsored through mental health funding provided by the Franklin/Fulton MH/IDD/EI Program, 425 Franklin Farm Lane, Chambersburg, PA 17202.

\section{Declarations}

Conflict of interest On behalf of all authors, the corresponding author states that there is no conflict of interest.

\section{References}

1. Acker GM. The challenges in providing services to clients with mental illness: managed care, burnout and somatic symptoms among social workers. Commun Ment Health J. 2010;4:591-600. https://doi.org/10.1007/s10597-0099269-5.

2. Atkinson DM, Rodman JL, Thuras PD, Shiroma PR, Lim KO. Examining burnout, depression, and self-compassion in veterans affairs mental health staff. J Alter Complement Med. 2017;23:551-7. https://doi.org/10.1089/acm.2017. 0087.

3. Bracke P, Chistiaens W, Verhaeghe M. Self-esteem, selfefficacy, and the balance of peer support among persons with chronic mental health problems. J Appl Soc Psychol. 2008;38:436-59.

4. Chinman M, George P, Dougherty RH, Daniels AS, Ghose SS, Swift A, Delphin-Rittmon ME. Peer support services for individuals with serious mental illnesses: assessing the evidence. Psychiatr Serv. 2014;65:429-41. https://doi.org/ 10.1176/appi.ps.201300244.
5. Corrigan PW, Holmes EP, Luchins D, Buican B, Basit A, Parks JJ. Staff burnout in a psychiatric hospital: a crosslagged panel design. J Organ Behav. 1994;15(1):65-74. https://doi.org/10.1002/job.4030150107.

6. Eriksson T, Germundsjö L, Åström E, Rönnlund M. Mindful self-compassion training reduces stress and burnout symptoms among practicing psychologists: a randomized controlled trial of a brief web-based intervention. Front Psychol. 2018. https://doi.org/10.3389/fpsyg.2018.02340.

7. Firmin RL, Mao S, Bellamy CD, Davidson L. Peer support specialists' experiences of microaggressions. Psychol Serv. 2019;16(3):456-62. https://doi.org/10.1037/ser0000297.

8. Gates LB, Akabas SH. Developing strategies to integrate peer providers into the staff of mental health agencies. Administr Policy Mental Health Mental Health Serv Res. 2007;34(3):293-306. https://doi.org/10.1007/s10488-0060109-4.

9. Jacobson N, Trojanowski L, Dewa CS. What do peer support workers do? A job description. BMC Health Serv Res. 2012;12:205. https://doi.org/10.1186/1472-6963-12-205.

10. Johnson J, Hall LH, Berzins K, Baker J, Melling K, Thompson C. Mental healthcare staff well-being and burnout: a narrative review of trends, causes, implications, and recommendations for future interventions. Int J Ment Health Nurs. 2018;27(1):20-32. https://doi.org/10.1111/ inm. 12416.

11. Landers GM, Zhou M. An analysis of relationships among peer support, psychiatric hospitalization, and crisis stabilization. Community Ment Health J. 2011;47(1):106-12. https://doi.org/10.1007/s10597-009-9218-3.

12. Kass R, Raftery A. Bayes Factors. J Am Stat Assoc. 1995;90(430):773-95. https://doi.org/10.2307/2291091.

13. Koutsimani P, Montgomery A, Georganta K. The relationship between burnout, depression, and anxiety: a systematic review and meta-analysis. Front Psychol. 2019. https://doi. org/10.3389/fpsyg.2019.00284.

14. Kraus SW, Stein CH. Recovery-oriented services for individuals with mentalillness and case managers' experience of professional burnout. Commun Ment Health J. 2013;49(1):7-13. https://doi.org/10.1007/s10597-0129505-2.

15. Leiter MP, Maslach C. Areas of worklife survey. California: Mind Garden Inc; 2000.

16. Leiter MP, Maslach C. Areas of worklife survey manual. 5th ed. California: Mind Garden Inc; 2011.

17. Li (Jessica) H, Yu K, Huang Y, Jin X. Not all leaving is created equal: Differentiating the factors of organizational and occupational turnover intentions. J Person Psychol. 2019;18(1):10-22. https://doi.org/10.1027/1866-5888/ a000216

18. Mancini MA, Lawson HA. Facilitating positive emotional labor in peer-providers of mental health services. Adm Soc Work. 2009;33(1):3-22. https://doi.org/10.1080/ 03643100802508619 .

19. Maslach C, Jackson SE. Maslach burnout inventory- human services survey. California: Mind Garden Inc; 1981.

20. Maslach C, Jackson SE, Leiter MP. Maslach Burnout Inventory. Fourth Edition. Mind Garden, Inc; 2018.

21. Moran GS, Russinova Z, Gidugu V, Gagne C. Challenges experienced by paid peer providers in mental health recovery: A qualitative study. Commun Ment Health J. 
2013;49(3):281-91. https://doi.org/10.1007/s10597-0129541-y.

22. Moran GS, Russinova Z, Gidugu V, Yim JY, Sprague C. Benefits and mechanisms of recovery among peer providers with psychiatric illnesses. Qual Health Res. 2012;22(3):304-19. https://doi.org/10.1177/ 1049732311420578.

23. Morse G, Salyers MP, Rollins AL, Monroe-DeVita M, Pfahler C. Burnout in mental health services: a review of the problem and its remediation. Administr Policy Mental Health Mental Health Serv Res. 2012;39(5):341-52. https:// doi.org/10.1007/s10488-011-0352-1.

24. Myrick K, del Vecchio P. Peer support services in the behavioral healthcare workforce: state of the field. Psychiatr Rehabil J. 2016;39(3):197-203. https://doi.org/10.1037/ prj0000188.

25. Neff KD. The development and validation of a scale to measure self-compassion. Self Identity. 2003;2(3):223-50. https://doi.org/10.1080/15298860309027.

26. Neff KD, Knox MC, Long P, Gregory K. Caring for others without losing yourself: an adaptation of the mindful selfcompassion program for healthcare communities. J Clin Psychol. 2020;76(9):1543-62. https://doi.org/10.1002/jclp. 23007.

27. O'Connor K, Neff DM, Pitman S. Burnout in mental health professionals: a systematic review and meta-analysis of prevalence and determinants. Eur Psychiatry. 2018;53:74-99. https://doi.org/10.1016/j.eurpsy.2018.06. 003.

28. Paris M Jr, Hoge MA. Burnout in the mental health workforce: A review. J Behav Health Serv Res. 2010;37(4):519-28. https://doi.org/10.1007/s11414-0099202-2.

29. Park SG, Chang B-H, Mueller L, Resnick SG, Eisen SV. Predictors of employment burnout among VHA peer support specialists. Psychiatr Serv. 2016;67(10):1109-15. https://doi.org/10.1176/appi.ps.201500239.

30. Peterson U, Demerouti E, Bergström G, Samuelsson M, Åsberg M, Nygren $\AA$. Burnout and physical and mental health among Swedish healthcare workers. J Adv Nurs. 2008;62(1):84-95. https://doi.org/10.1111/j.1365-2648. 2007.04580.x.

31. Raes F, Pommier E, Neff KD, Van Gucht D. Construction and factorialvalidation of a short form of the Self-Compassion Scale. Clin Psychol Psychother. 2011;18(3):250-5. https://doi.org/10.1002/cpp.702.
32. Salyers MP, Fukui S, Rollins AL, Firmin R, Gearhart T, Noll JP, Williams S, Davis CJ. Burnout and self-reported quality of care in community mental health. Administr Policy Ment Health Ment Health Serv Res. 2015;42(1):61-9. https://doi.org/10.1007/s10488-0140544-6.

33. Scanlan JN, Still M, Radican J, Henkel D, Heffernan T, Farrugia P, Isbester J, English J. Workplace experiences of mental health consumer peer workers in New South Wales, Australia: A survey study exploring job satisfaction, burnout and turnover intention. BMC Psychiatry. 2020. https:// doi.org/10.1186/s12888-020-02688-9.

34. Sklar M, Ehrhart MG, Aarons GA. COVID-related work changes, burnout, and turnover intentions in mental health providers: A moderated mediation analysis. Psychiatr Rehabil J. 2021. https://doi.org/10.1037/prj0000480.

35. Sells D, Davidson L, Jewell C, Falzer P, Rowe M. The treatment relationship in peer-based and regular case management for clients with severe mental illness. Psychiatr Serv. 2006;57:1179-84. https://doi.org/10.1176/appi.ps.57. 8.1179

36. Sledge WH, Lawless M, Sells D, Wieland M, O’Connell MJ, Davidson L. Effectiveness of peer support in reducing readmissions of persons with multiple psychiatric hospitalizations. Psychiatr Serv. 2011;62(5):541-4. https://doi. org/10.1176/appi.ps.62.5.541.

37. Substance Abuse and Mental Health Services Administration (2012). SAMHSA's Working Definition of Recovery. PEP12-RECDEF, Rockville, MD: Center for Mental Health Services, Substance Abuse and Mental Health Services Administration, U.S. Department of Health and Human Services.

38. Tartakovsky E, Gafter-Shor A, Perelman-Hayim M. Staff members of community services for people with intellectual disability and severe mental illness: Values, attitudes, and burnout. Res Dev Disabil. 2013;34(11):3807-21. https:// doi.org/10.1016/j.ridd.2013.08.026.

39. Yanchus NJ, Periard D, Osatuke K. Further examination of predictors of turnover intention among mental health professionals. J Psychiatr Ment Health Nurs. 2017;24(1):41-56. https://doi.org/10.1111/jpm.12354.

Publisher's Note Springer Nature remains neutral with regard to jurisdictional claims in published maps and institutional affiliations. 